\title{
SEXTUPOLE CORRECTION MAGNETS FOR THE LARGE HADRON COLLIDER
}

\author{
R. B. Meinke ${ }^{\#}$, W. M. Hinson, M. Senti, Advanced Magnet Lab, Inc. Palm Bay, FL \\ W.J. Op de Beeck, C. De Ryck, SMCE NV, Heist-op-den Berg, Belgium
}

\section{Abstract}

About 2,500 superconducting sextupole corrector magnets (MCS) are needed for the Large Hadron Collider (LHC) at CERN to compensate persistent current sextupole fields of the main dipoles. The MCS is a cold bore magnet with iron yoke. The coils are made from a NbTi conductor, which is cooled to $1.9 \mathrm{~K}$. In the original CERN design 6 individual sub-coils, made from a monolithic composite conductor, are assembled and spliced together to form the sextupole. The coils are individually wound around precision-machined central islands and stabilized with matching saddle pieces at both ends. The Advanced Magnet Lab, Inc. (AML) has produced an alternative design, which gives improved performance and reliability at reduced manufacturing cost. In the AML design, the magnet consists of three splice-free sub-coils, which are placed with an automated winding process into pockets of prefabricated G-11 support cylinders. Any assembly process of sub-coils with potential misalignment is eliminated. The AML magnet uses a Kapton-wrapped mini-cable, which allows helium penetration into the vicinity of the conductor, increasing its cryogenic stability. Eliminating all internal splices from the magnet significantly reduces heat loads and the risk of magnet failure during operation. A tested prototype reached the critical current limit of the conductor in the first quench.

\section{INTRODUCTION}

In the LHC, currently under construction at CERN, each bore of the main bending dipole is equipped with a superconducting sextupole (MCS) corrector magnet to enable compensation of systematic sextupole errors in the dipole field. About 2500 sextupole magnets of this type are needed for the accelerator.

In the original CERN design [1] the MCS magnet consists of six coils, which are individually wound and then assembled and spliced to form the required sextupole. Twelve MCS prototypes of this kind have been manufactured and tested. All magnets showed significant training behavior at $1.9 \mathrm{~K}$ and unacceptable heat loads from the conductor splices [2].

This paper describes an alternative design of the MCS sextupole developed by the AML under contract of

"Email: rmeinke@magnetlab.com
CERN. The sextupole has significantly improved quench performance, uses stronger insulation to avoid electrical shorts in the coil, eliminates all internal splices in the magnet, and uses an automated coil winding process to guarantee reliable mass production at a minimum manufacturing cost. Key features of the new design and results form the first tests are presented in this paper.

\section{SEXTUPOLE DESIGN}

\subsection{Original CERN Design}

The key parameters of the MCS sextupole are summarized in Table 1 [1].

Table 1: Main parameters of MCS sextupole

\begin{tabular}{|l|c|c|}
\hline \multicolumn{1}{|c|}{ Item } & Unit & Value \\
\hline Field gradient & $\mathrm{T} / \mathrm{m}^{2}$ & 1657 \\
\hline Integrated field at $10 \mathrm{~mm}$ & $\mathrm{~T} \mathrm{~m}$ & 0.0187 \\
\hline Nominal current & $\mathrm{A}$ & 550 \\
\hline Effective length & $\mathrm{mm}$ & 113 \\
\hline Overall Length & $\mathrm{mm}$ & 160 \\
\hline Bore of magnet & $\mathrm{mm}$ & 60 \\
\hline Operating temperature & $\mathrm{K}$ & 1.9 \\
\hline
\end{tabular}

The sextupole consists of six sub-coils, each occupying an exact $60^{\circ}$ cylindrical sector. The individual coils are wound as double pancake coils around a precisely machined central G-11 island (see Figure 1). At the two ends of the coils, G-11 saddle pieces are placed to impart rigidity and to serve as a support for the lead end connections.

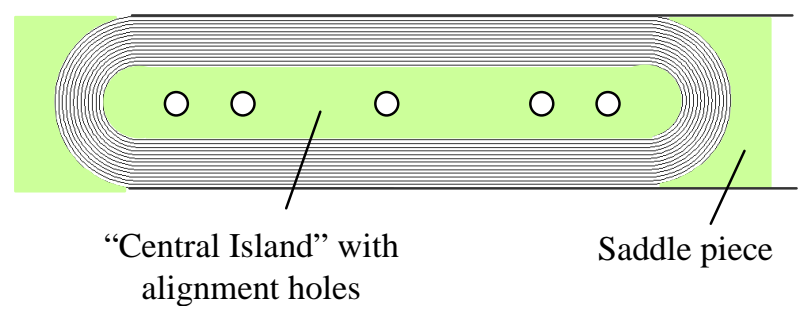

Figure 1: Sketch of individual sextupole coil of original CERN design.

A rectangular $\mathrm{NbTi}$ conductor with a $\mathrm{Cu} / \mathrm{SC}$ ratio of 1.6 and bare dimensions of $1.13 \times 0.61 \mathrm{~mm}^{2}$ is used for the coils. The conductor is insulated with thin layer $(0.06$ $\pm 0.01 \mathrm{~mm}$ ) of Polyester-imide. The coils are wet wound and then cured at a slightly elevated temperature. 
Six coils are aligned on a mounting fixture and glued together at their mating faces to form the sextupole. A premachined tube of insulating material is glued over this assembly. The conductors from the individual coils are spliced together to achieve a series connection of the six coils. The whole assembly is surrounded by a laminated soft iron yoke and inserted in an aluminum shrink cylinder.

\subsection{AML Sextupole Design}

Under contract of CERN the Advanced Magnet Lab, Inc. in Palm Bay Florida has developed an alternative design of the MCS sextupole and produced two prototype magnets.

The coils are wound from a $\mathrm{NbTi}$ round mini-cable with 6-around-1 strands. Important parameters of the used conductor are summarized in Table 2.

Table 2: Superconducting cable parameters

\begin{tabular}{|l|c|c|}
\hline \multicolumn{1}{|c|}{ Item } & Unit & Value \\
\hline Number of wires & & 7 \\
\hline Wire diameter & $\mathrm{mm}$ & 0.33 \\
\hline Filament diameter & $\mu \mathrm{m}$ & 10 \\
\hline \# filaments / wire & & 310 \\
\hline Cu/SC ratio & & $2.5 \pm 0.1$ \\
\hline Twist pitch & Twist $/ \mathrm{cm}$ & 1.06 \\
\hline Diameter bare & $\mathrm{mm}$ & 0.99 \\
\hline Diameter insulated & $\mathrm{mm}$ & 1.07 \\
\hline Icrit at 5 T, 4.2 K & $\mathrm{A}$ & 476 \\
\hline
\end{tabular}

In contrast to a conductor with rectangular cross section, the round cable can be easily bent in any direction. This feature significantly simplifies the winding process, and in particular facilitates the manufacturing of coil ends. A rectangular conductor requires complex, constantperimeter conductor arrangements, which require significant design and manufacturing effort.

The mini-cable is wrapped with Kapton foil $(50 \%$ overlap), which forms an insulating sleeve around the conductor. The Kapton is mechanically stronger than varnish insulation and the probability of an electrical short in the coil is significantly reduced. Experience with Kapton has shown that slight movements of the conductor inside the insulation do not trigger a quench of the superconductor. Furthermore, helium can penetrate into the Kapton sleeve and make direct contact with the superconductor. It therefore takes a significantly higher energy deposition to trigger a quench in such a coil.

\subsection{Coil Design}

The conductor layout of the AML coil with the sub-coil interconnections is shown in Figure 2. Each sub-coil has two layers; the inner layer is wound from the outside towards the inside of the coil and the outer layer from the inside back to the outside.

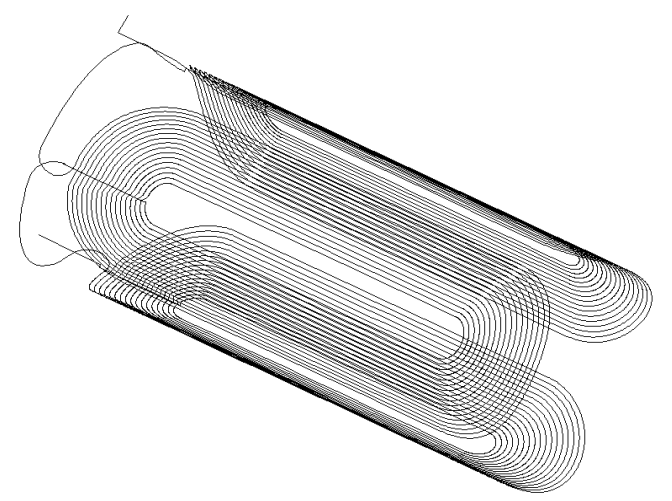

Figure 2: Coil cross section of AML designed MCS coil. For reasons of clarity only one conductor layer is shown.

A fiber-reinforced tube with the required inner magnet diameter is used as a coil support. Pockets are machined or molded into this tube to accommodate 3 sub-coils that form the sextupole magnet. The outer diameter of the support tube is chosen to be flush with the conductor of the inserted coils. The bottom of the pockets have a wall thickness of about $0.5 \mathrm{~mm}$ to guarantee good mechanical stability of the coil assembly (see Figure 3).

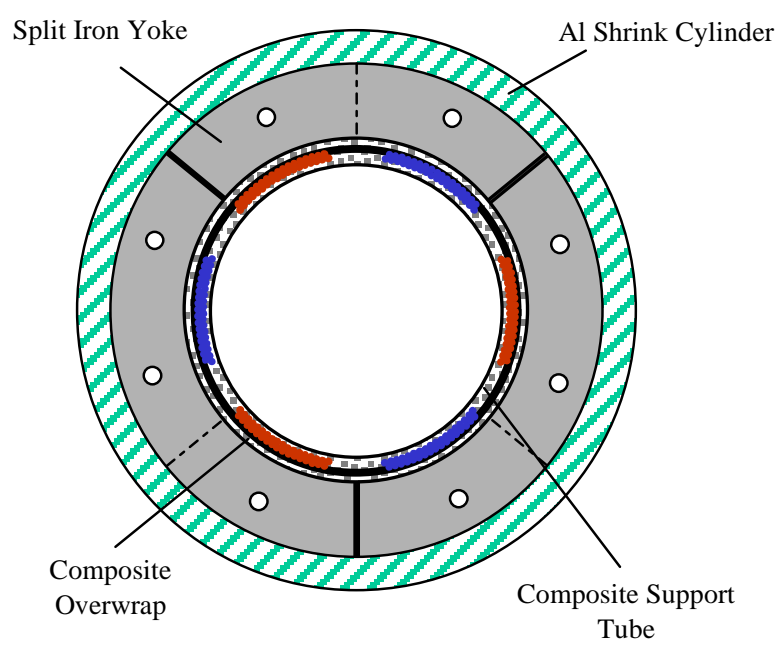

Figure 3: Cross section of MCS designed by AML.

The coils are directly wound into the machined pockets of the support tube, using AML's "Direct Adhesive" coil winding technology. In this winding process the conductor 
is precisely placed with the help of a multi-axis robot and instantaneously bonded to the support structure. Adhesive for conductor bonding inside of the coil is automatically added during the winding process. The coils are wound with a continuous conductor; the leads from one sub-coil to the next are placed and stabilized in grooves of the support tube.

The common support tube for the three sub-coils and the continuous winding process, automatically yield a precise alignment of the individual sub-coils relative to each other.

Remaining gaps between the coils and the walls of the support tube pockets are filled with epoxy putty to avoid any movement of the conductor during current excitation. The support tube with the inserted coils is over-wrapped with epoxy impregnated fiberglass and cured at an elevated temperature. The result is a precise, very stable coil assembly, which contains all required interconnections. AML used its own adhesive systems for coil winding. Standard cryogenic tests [3] of the CERN proposed epoxy-resin system, AW 106 and HV 953 U Hardener from CIBA, showed severe cracking in the first shock cycle, while 25 cycles without cracking are recommended.

The outer surface of the overwrap is machined to precisely fit the laminations of the surrounding soft iron yoke (see Figure 3).

The laminations are divided into $120^{\circ}$ sections, which leave small gaps between them. These gaps close when the magnet is cooled down and the outer aluminum shrink cylinder puts the coil assembly under radial pressure.

\section{TEST RESULTS}

The first test coil without iron yoke, but equipped with an aluminum shrink cylinder underwent quench tests at CERN. Three test series at different temperatures were performed with warm-up cycles of the coil between tests. The test results are shown in Figure 4.

The coil reached the critical current of the conductor in the first quench and shows no significant variation in quench current thereafter. The current ramp rate was 10 $\mathrm{A} / \mathrm{sec}$ throughout the tests.

The second magnet, which was equipped with an iron yoke, showed unexplained ramp rate dependence in a preliminary test and will be retested.

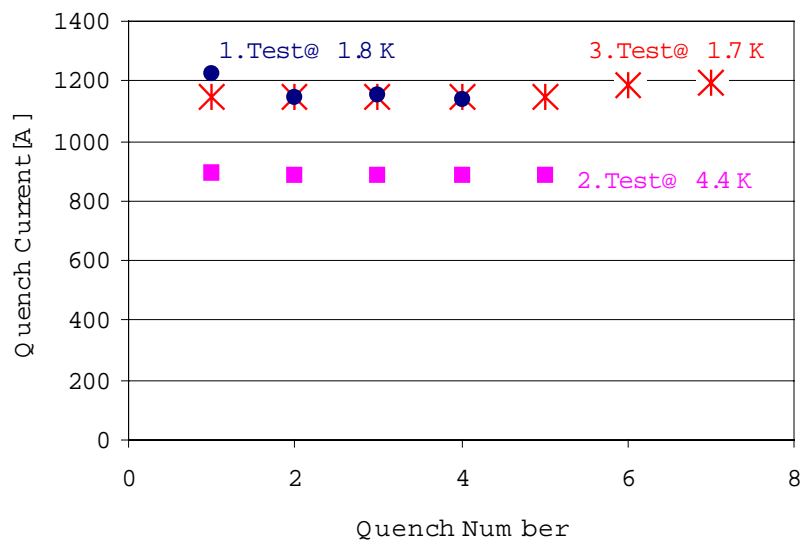

Figure 4: Quench test results of 1 test coil.

\section{SUMMARY}

The MCS sextupole magnet, designed by AML for the LHC project, offers the following improvements over the original CERN design. The used mini-cable offers better quench performance since each conductor strand is surrounded by helium, which penetrates into the Kapton insulation surrounding the cable. The round, flexible cable simplifies the coil ends, can be used in an automatic winding process, and the redundancy of conductors in the cable improves the reliability of the magnets.

The proposed design eliminates more than 12,500 splices in LHC for the sextupoles alone and solves the heat load problem of the previous CERN design. The applied coil manufacturing technology significantly simplifies the magnet assembly process by eliminating any sub-coil alignment and assembly process.

The new AML design offers magnets with improved quench performance, reduced heat loads, and better operational reliability. The simplified manufacturing process and the automated coil winding reduce the overall cost of these magnets. The proposed design and manufacturing technology is directly applicable to the more than 2,500 decapoles needed for LHC.

\section{REFERENCES}

[1] Technical Specification of the Sextupole Corrector Magnet", CERN/LHC-ICP/JS/98-001.

[2] J. Salminen et al. "Experience with the Fabrication and Testing of the Sextupole Superconducting Corrector Magnets for the LHC", MT-15 Proceedings, Oct. 1997.

[3] A.B. Brennan et al, "Epoxy Materials for Improved Performance at $4 \mathrm{~K}$ ", Proceedings of the International Workshop "High Magnetic Fields: Applications, Generation, Materials, 1996. 